\title{
RFID Medication Management System in Hospitals
}

\author{
https://doi.org/10.3991/ijoe.v16i12.17031 \\ Safae El Abkari $\left.{ }^{\bowtie}\right)$, Abdelilah Jilbab, Jamal El Mhamdi \\ Mohammed V University, Rabat, Morocco \\ safae.abkari@um5s.net.ma
}

\begin{abstract}
One of the primary concerns of the World Health Organization is the improvement of medical care by reducing adverse events in the medication process and enhancing the safety of patients. These issues are mainly related to the management of expensive and high-risk medicines in hospitals. In this paper, we enhanced medication management by minimizing the possibility of medication errors from its prescription-validation to its preparation. For this purpose, we designed a hospital and pharmacy services management system by employing digital signature using Radio Frequency IDentification technology. Our proposed system is equipped with ESP8266 modules, RFID readers and tags which allow the detection of taking medications and ensures an automated medication management.
\end{abstract}

Keywords—Radio frequency IDentification, medication, safety, traceability, patient safety.

\section{Introduction}

Recently, information technologies are very used in various economical services. Healthcare field is mainly linked to the economic development [1]. It is rapidly evolving which causes high needs for efficient hospital management applications [2], [3]. The process of modernization of healthcare institutions is undergoing leading to various innovational health management methods. In hospitals, the occurrence percentage of medication error for ordering is $56 \%$, administration is $34 \%$, transcribing is $6 \%$ and $4 \%$ for dispensing [4], [5]. Although hospital statistical data show that most healthcare institutions have computerized medical data, the Institute of Medicine (IOM) reported that a patient can be subjected to multiple medication error during the clinical process [6]. In addition, each year the United States have approximatively 1.5 million of preventable medication errors. Hospital management comes a solution to speed up the material and information flow, improve the medication safety and reduce errors [7], [8]. Similar system can also be used for recording the health cards of patients.

Latest European Union guidelines and trends have established the introduction and the implementation of hospital management standards. In 2019, the European Union countries introduced medication labeling standards and by the end of 2020 instruments, biological samples, and medical devices will be labeled. Therefore, automatic 
identification elements have come under the spotlight and became a trend. Thus, it is necessary to determine the correct identification technology depending on the application context.

In healthcare environment, it is necessary to develop the following actions to ensure:

- Reduce medication errors by preventing and identifying adverse events.

- Evaluate and fix medication errors when they occur.

Traditionally, medical institutions use the managing method of warehouses [9], [10]. In warehouses, different types of method are implemented to assign data to products in logistics [11]. For example, according to the regulation of medicine labeling, the European Union medicines and materials are labelled with a European Article Number (13-digit code), a serial number, a batch number, and a Global Trade Identification Number. These data are presented on the packaging of the product in a GS1 code format. The matrix code of GS1 data is read when medications are issued in order to verify and confirm the action. In case the medication genuineness is confirmed, the medications are given to the patient.

Radio Frequency IDentification (RFID) technology is one of the most used solution for people an assets identification. Thus, it is a promising technology for automatic generation of product documentation, inventory management, supply chain, online tracking [12], [13] and healthcare applications [14].

The main contributions presented in this paper are the following:

- A hospital and Pharmacy service management system using digital signature with Radio Frequency IDentification technology.

- A speed up of the material and information flow and improvement of medication safety with an automated and intelligent medication management.

In the following paper, we presented related works in Section 2. We introduced our proposed system and presented its performances in Section 3. Finally, conclusion is given in Section 4.

\section{Related Works}

Different researches on medication management systems have been developed and achieved high performances by adopting the appropriate designation technology. Table below presents different existing management systems. 
Table 1. Survey of management systems

\begin{tabular}{|l|l|}
\hline Ref. & \multicolumn{1}{|c|}{ Description } \\
\hline$[15]$ & $\begin{array}{l}\text { Proposed a framework for personalized medication treatment management. } \\
\text { Could be employed to extend the existing personalized care systems. }\end{array}$ \\
\hline$[16]$ & $\begin{array}{l}\text { Enhanced inpatient medication safety by proposing a double RFID based system. } \\
\text { Proposed an extended RFID authentication protocol for online cases and RFID grouping proof for } \\
\text { offline cases. }\end{array}$ \\
\hline$[17]$ & $\begin{array}{l}\text { Proposed a hardware architecture and technical scheme of an intelligent information system based } \\
\text { on cloud computing and IoT. } \\
\text { The architecture of the system is constituted of: network, three layers of perception, and application. } \\
\text { Improved the efficiency among the systems by realizing a communication and an interconnection } \\
\text { among communication terminals. }\end{array}$ \\
\hline$[18]$ & $\begin{array}{l}\text { Proposed an indoor positioning system based on wireless signals. } \\
\text { Improved the accuracy and the reliability of the collected data using P-P plot, Q-Q plot, and Gaussi- } \\
\text { an distribution. }\end{array}$ \\
\hline$[19]$ & $\begin{array}{l}\text { Evaluated the application of RFID technology in tracing medications and patients in an intensive- } \\
\text { care unit. } \\
\text { Demonstrated the technical viability of RFID for the medicine process and concluded that the appli- } \\
\text { cation cost was too high. }\end{array}$ \\
\hline$[20]$ & $\begin{array}{l}\text { Obtained the traceability of medication and patients using RFID technology. } \\
\text { Studied the effectiveness in the routine hospital clinical practice for health personnel. }\end{array}$ \\
\hline$[21]$ & $\begin{array}{l}\text { Proposed an automated monitoring system for medication stocks. } \\
\text { Used two types of sensors which ensure a wide range of medical product management (medicines } \\
\text { and medical equipment). }\end{array}$ \\
\hline
\end{tabular}

\section{Our Proposed Management System}

\subsection{Radio frequency IDentification technology}

Radio Frequency IDentification [22] was originally developed along with the development of mobile telephones, computers, and wireless networks. It is implemented in different environments such as healthcare to avoid counterfeiting of medicines. The process of identification (Fig.1.) consists of reading an RFID tag attached to an asset or a person without any physical contact.

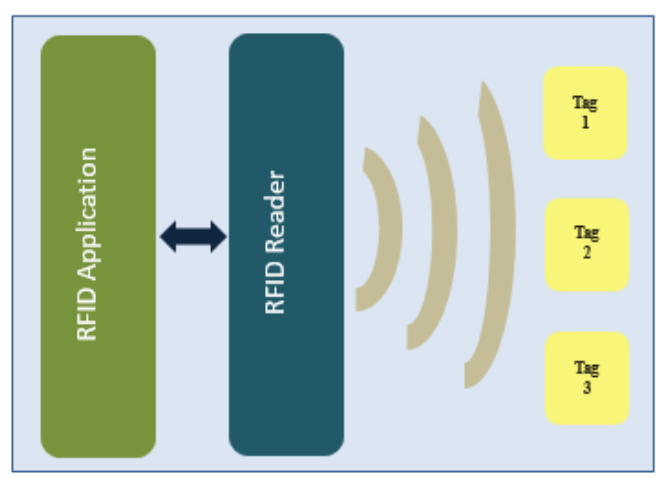

Fig. 1. RFID identification process 
If the tag possesses the same energy source as the reader, then it can be classified as passive. If the tag does not possess the same energy source as the reader, then this tag is active. The third category is the semi-active tags wherein the tag energy source is used for power supplying the chip and the reader energy source is used for data transmission.

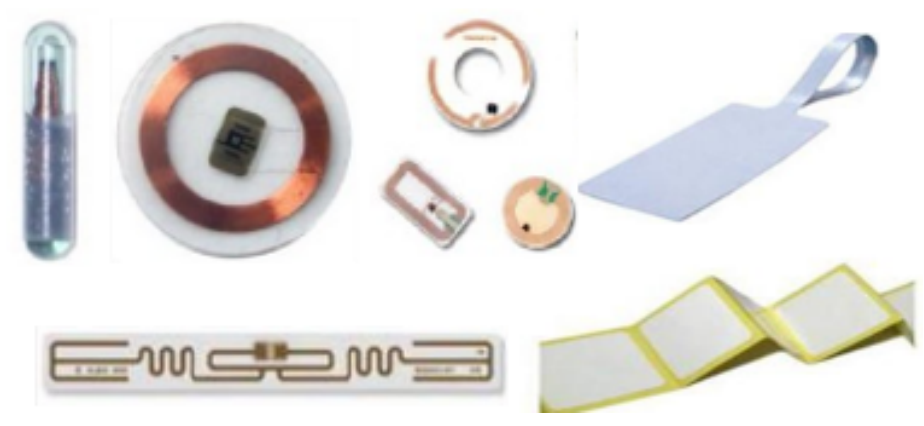

Fig. 2. RFID tags

RFID technology works under the following frequency bands:

- Low Frequency: $125-134 \mathrm{KHz}$

- High Frequency: $13.6 \mathrm{MHz}$

- Ultra-High Frequency: 865-954 MHz

- Microwave: $2.4-5.8 \mathrm{GHz}$

RFID technology offers large possibilities such as different shapes of tags and storing information in the tag memory but requires extensive research due to its particular complexity.

\subsection{Methodology and results}

In order to design an effective medicine management system, it is necessary to select the right components that will operate with the medicine process (ordering, administration, transcribing and dispensing). The main components of our proposed system are the following:

- Arduino Uno board

- ESP8266 modules

- RFID readers (NFC)

- Passive RFID tags

- Computer 
Table 2. Component of the proposed system

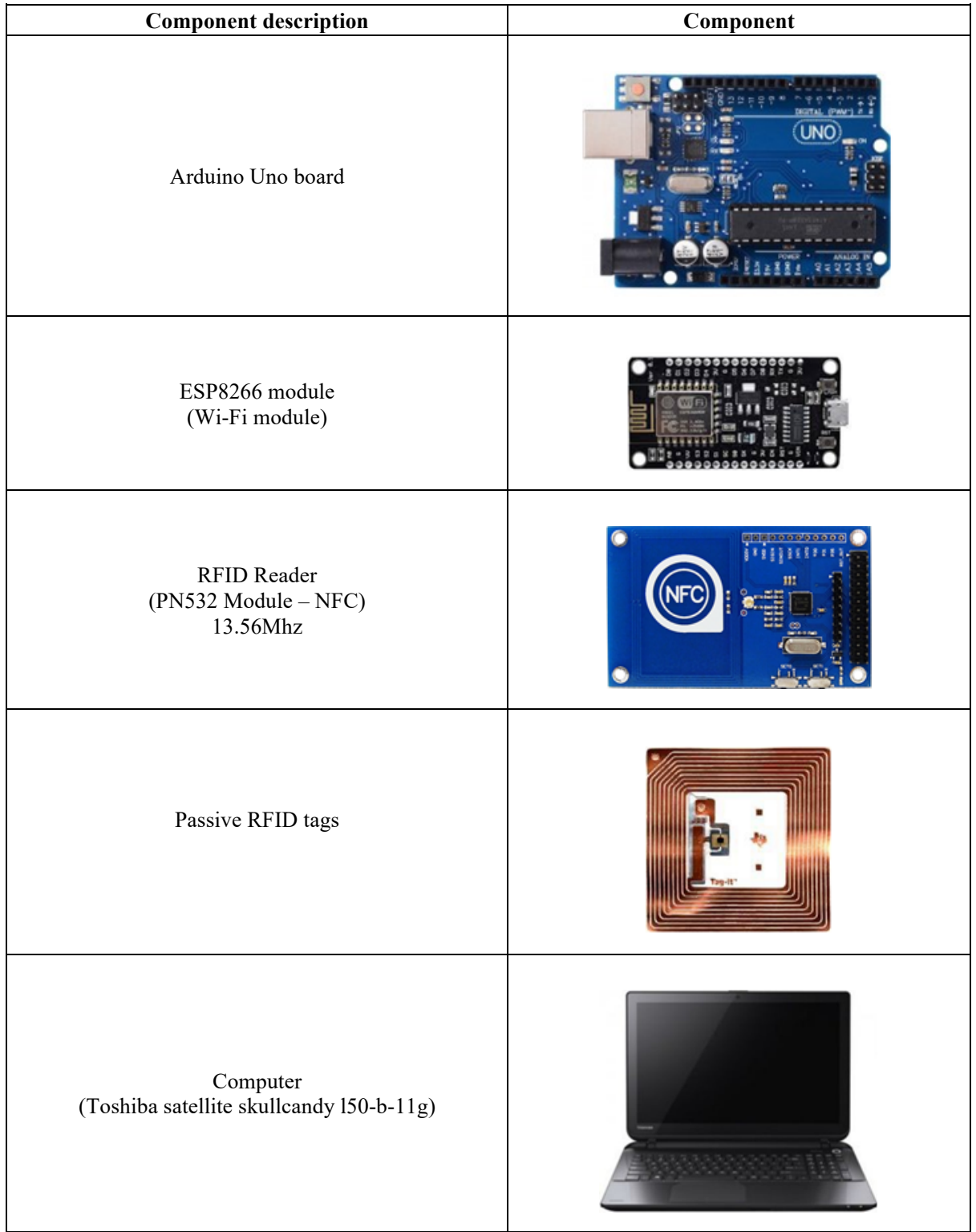

Our proposed management system is constituted of three main parts: the pharmacy system (Fig.2.) which is placed in the pharmacy, the doctor/nurse modules (Fig.3.) which are carried by the medical personnel and the tracking modules (Fig.3.) which is placed at each hospital room gate. All medications, doctors, nurses and patients have RFID tags and are identified by PN532 NFC modules. These RFID tags do not need any physical contact with RFID readers. The communication between RFID module 
and Arduino Uno board of the pharmacy system is made via Serial Peripheral Interface (SPI) using SS, clock, MISO and MOSI). Arduino Uno board and ESP8266 communication is made via TX and RX for the other modules. ESP8266 modules are used to send RFID ID to the pharmacy system to track medications. The system needs a power supply of only $3.3 \mathrm{~V}$.

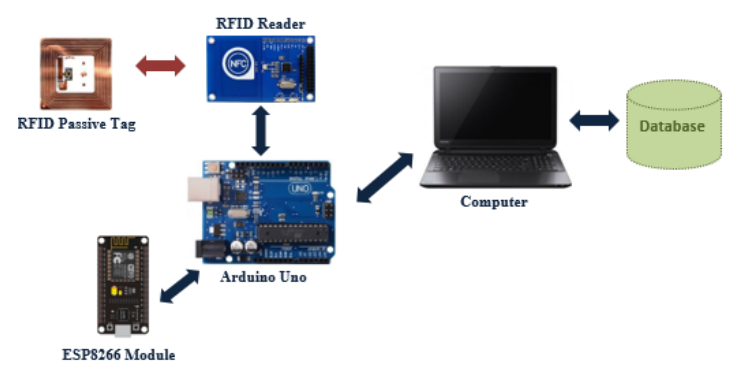

(a)

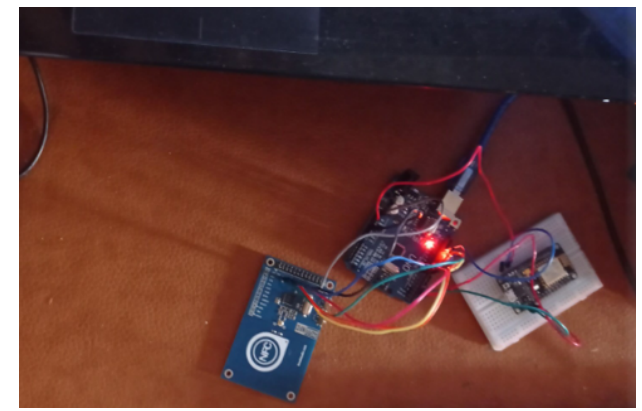

(b)

Fig. 3. (a) The design of the pharmacy system (b) our proposed pharmacy system

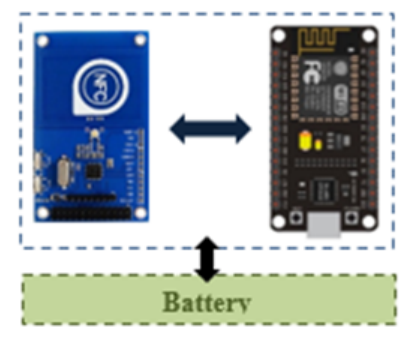

(a)

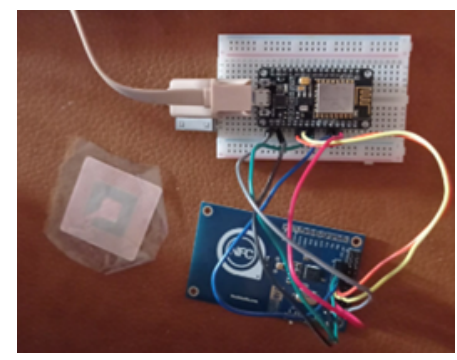

(b)

Fig. 4. (a) The tracking and the nurse/doctor module design (b) our proposed tracking and nurse/doctor module 


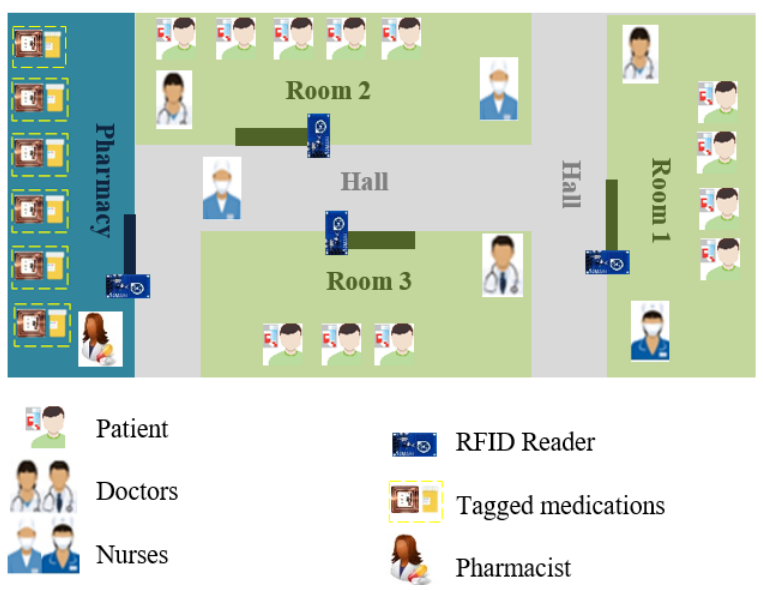

Fig. 5. Placement of the management system in the hospital

Each medication was assigned by a digital signature (RFID tag attached to the medication packaging back) to its unique identification code (ID). When a medication is transported by a nurse through RFID gates, the medication is identified and tracked by comparing its stored digital signature and its ID.

Table 3. Medications with their respective ID codes

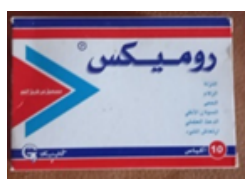

ID: C5 38 1F 5A

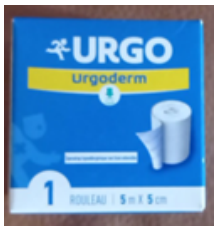

ID: C5 34 2B 14

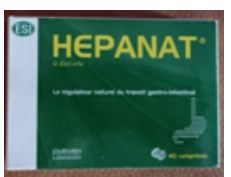

ID: C5 $382 \mathrm{~F} 6 \mathrm{E}$

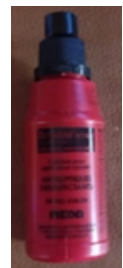

ID: C5 31 5F 38

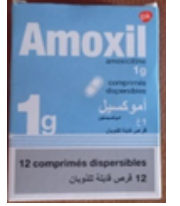

ID: C5 37 3F 5C

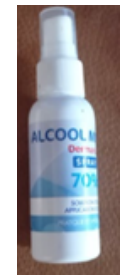

ID: C5 362 F 99

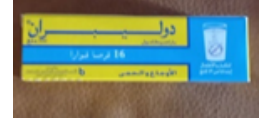

ID: C5 39 FF 67

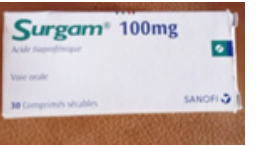

ID: C5 38 3B 45

Each doctor, nurse and patient have a unique digital signature which is its unique RFID identification code (ID). 
Table 4. Doctor, nurse and patient with their respective ID codes

Nurses

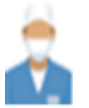

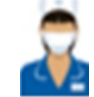

ID:EE EE EE 01
Doctors

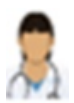

ID: AA AA AA 01

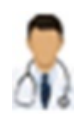

ID: BB BB BB 01
Patien

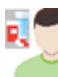

ID: DD DD DD 01

The process flow of the medication management system is described as follows:

- Step1: When a doctor prescribes medications after diagnosing a patient, data of the prescription, the patient and the nurse in charge are stored in the pharmacy system database using the doctor digital signature.

- Step 2: After the reception of the prescription, the system automatically assigns each medication to an RFID tag ID.

- Step 3: After checking and preparing the prescription, the pharmacist sends a notification to the nurse.

- Step 4: The nurse checks if there is any updates, changes or errors in the prescription. If not, the nurse confirms the reception of the right medications with her digital signature via a her/his mobile RFID reader.

- Step 5: The tracking system starts tracking and sends notifications of the medication locations.

- Step 6: The nurse dispenses medications to the patient.

- Step 7: If needed, the doctor updates the prescription of the medications.

The proposed medication process is divided into two main phases: Prescription phase and medicine dispensing phase.

\subsection{Prescription phase}

In this first stage, the doctor prescribes medications after diagnosing a patient. Once the doctor's ID is verified, the pharmacist prepares the medications and sends a notification to the nurse in charge. The doctor receives a query to change the prescription if the pharmacist suspects an error in the prescription. 


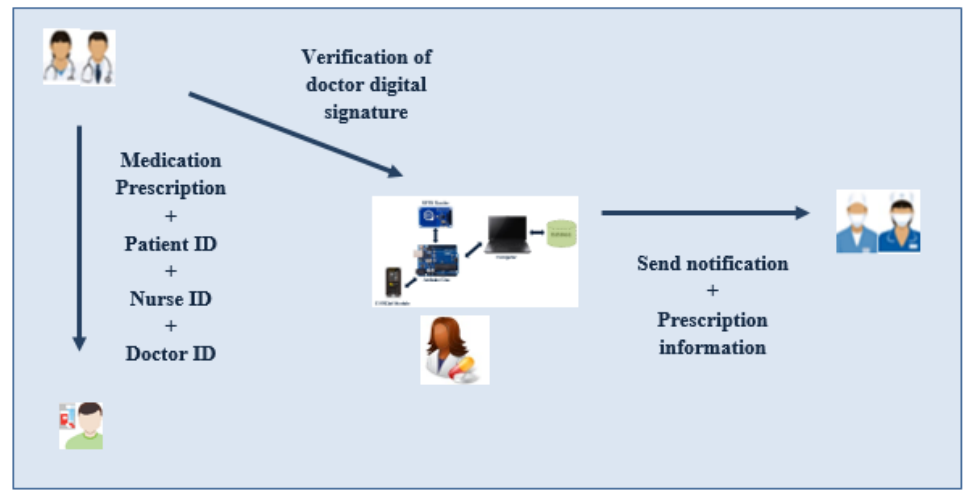

Fig. 6. Prescription phase overview

The prescription phase process can be described as follows:

- Step 1: The doctor sends its prescription along with her/his ID, the patient ID, and the nurse ID using her/his module. If the doctor ID is confirmed at the pharmacy system level. The pharmacist prepares the prescription by packing medications and scanning their respective RFID tags.

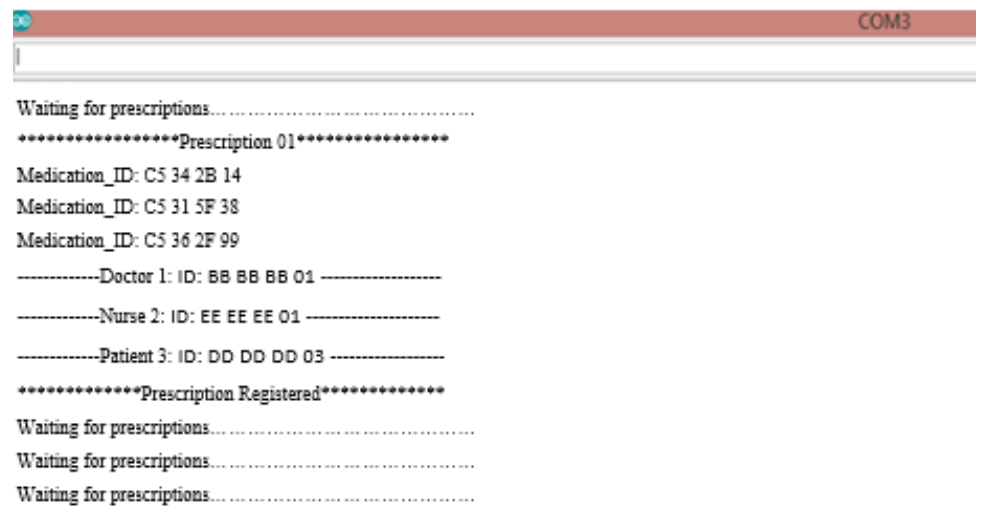

Fig. 7. Prescription sent by the doctor at the pharmacy system level

- Step 2: The pharmacist sends a notification to the nurse in charge of the patient medication.

\subsection{Dispensing medication phase}

In this last stage, the nurse verifies if the prescription is right (patient, medications, dosage and time are right). 


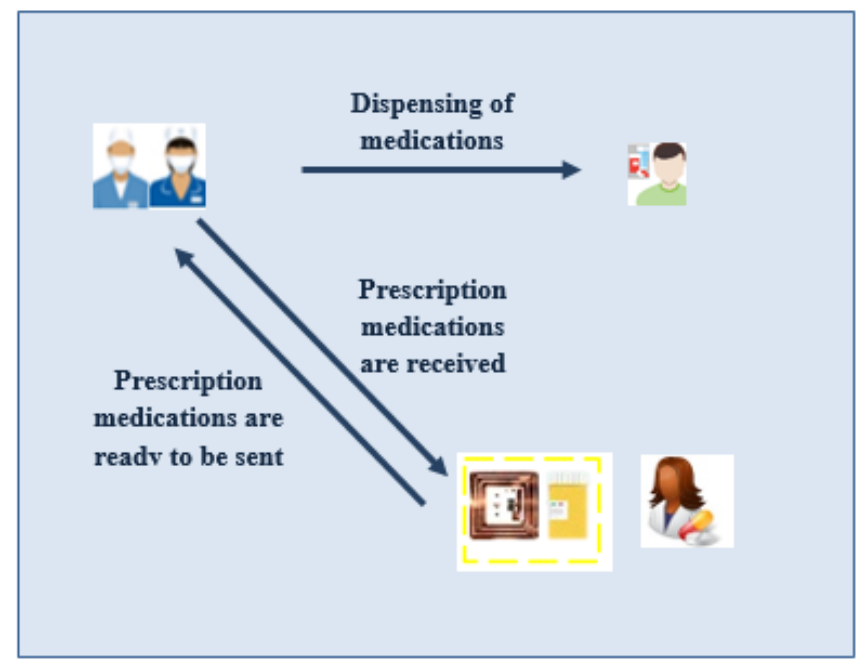

Fig. 8. Dispensing medication phase overview

The process of the dispensing of medications is as follows:

- Step 1: The nurse checks the prescription information. If the nurse suspects an error, she/he alerts the pharmacist using her/his module.

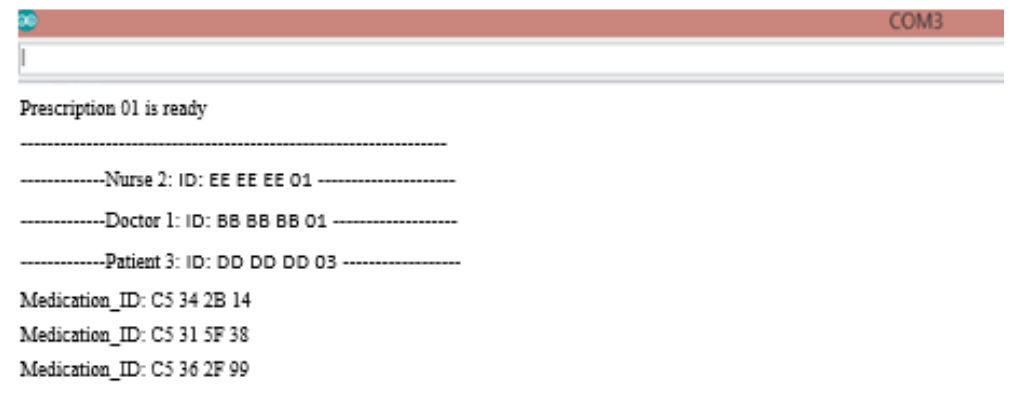

Fig. 9. Prescription information received by the nurse

- Step 2: If the information is right, the nurse receives medications and confirms the reception. 


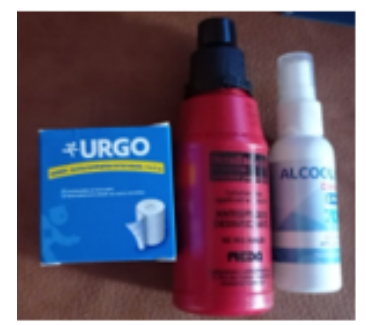

Fig. 10.Medications received by the nurse

- Step 3: The nurse dispenses medications to the patient and confirms the dispensing.

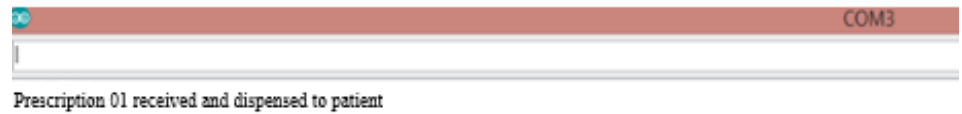

Fig. 11. Confirmation of the dispensing of medications

When the nurse receives the prescription prepared by the pharmacist, those medications are tracked in the hospital. The tracking modules send notifications of medication locations to the pharmacist system.

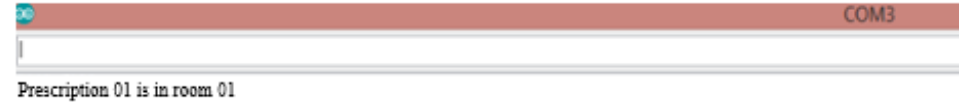

Fig. 12. Tracking notification sent by the tracking module to the pharmacy system

\subsection{Case of changes in the prescription}

If after taking medications the patient has any unusual reaction, the nurse notifies the doctor. The doctor then renews the prescription and goes to step 1 of the prescription phase.

Our proposed system demonstrates an improvement of the medication process and patient safety in hospitals. The advantages and disadvantages of this management system are the following:

Table 5. Advantages and Disadvantages of our proposed management system

\begin{tabular}{|l|l|}
\hline \multirow{5}{*}{ Advantage } & Immunity against environmental changes (temperature...) \\
& Security \\
Mobile object identification \\
Ease of use \\
Fast reading and scanning of multiple objects \\
Write/Rewrite ability \\
\hline The nurse/doctor modules can easily be replaced by smartphones. \\
\hline Disadvantage & Sensibility to environmental signals mainly caused by medical devices \\
& Need of professionals for the system technical support \\
\hline
\end{tabular}




\section{Conclusion}

Our proposed RFID medication management system experimentally demonstrated to be effective for the improvement of the medication process and patient safety. This system can be integrated into various medication planning and managing systems due to its simplicity of use and adaptability. Our proposition is also able to speed up the information flow, improve the medication-patient safety and eliminate errors caused by healthcare institutions' employees by scanning a large number of medications at the same time. Our RFID medication management system is designed according to the requirements of the recent healthcare trends. Thus, the nurse/doctor modules can easily be replaced by smartphones.

\section{$5 \quad$ Perspectives}

Besides the effectiveness of our medication management system, developing a management software and database also adding a display screen to the doctor/nurse module remain important issues we will focus on in our future works.

\section{Acknowledgement}

We are grateful to Pr. El Hassan El Abkari for insightful discussions.

\section{$7 \quad$ References}

[1] Balog, Michal, Jozef Husár, and Angelina Iakovets. "RFID System for Effective Managing of Medical Facilities." New Approaches in Management of Smart Manufacturing Systems. Springer, Cham, 2020. 1-23. https://doi.org/10.1007/978-3-030-40176-4 1

[2] Husar, J., and A. Iakovets. "Proposal for monitoring the movement of biological samples in hospitals." Automation and Management in Theory and Practice, 13th Anniversary Conference of Universities, High Schools and Practice, Stará Lesná, SR. 2019.

[3] Husar, J., and A. Iakovets. "Fourth industrial revolution technologies, management of manufacturing systems 2018." TUKE, FVT, Dubrovnik, Croatia (2018).

[4] Bates, David W., et al. "Incidence of adverse drug events and potential adverse drug events: implications for prevention." Jama 274.1 (1995): 29-34. https://doi.org/10.1001/ja $\underline{\text { ma.1995.03530010043033 }}$

[5] Committee on Identifying and Preventing Medication Errors. "Preventing medication errors: quality chasm series." (2007): 221-55. https://doi.org/10.17226/11623

[6] Omotosho, Adebayo, and Peace Ayegba. "Medication Adherence: A Review and Lessons for Developing Countries." International Journal of Online and Biomedical Engineering (iJOE) 15.11 (2019): 104-123. https://doi.org/10.3991/ijoe.v15i11.10647

[7] Adhikari, Radha, et al. "A multi-disciplinary approach to medication safety and the implication for nursing education and practice." Nurse education today 34.2 (2014): 185-190. https://doi.org/10.1016/j.nedt.2013.10.008 
[8] Koutkias, Vassilis G., et al. "A personalized framework for medication treatment management in chronic care." IEEE Transactions on Information Technology in Biomedicine 14.2 (2009): 464-472.

[9] Li, Jianqiang, et al. "PSOTrack: A RFID-based system for random moving objects tracking in unconstrained indoor environment." IEEE Internet of Things Journal 5.6 (2018): 46324641. https://doi.org/10.1109/jiot.2018.2795893

[10] Tejesh, B. Sai Subrahmanya, and S. J. A. E. J. Neeraja. "Warehouse inventory management system using IoT and open source framework." Alexandria engineering journal 57.4 (2018): 3817-3823. https://doi.org/10.1016/j.aej.2018.02.003

[11] Balog, M., D. Matiskova, and P. Semanco. "System of Workpiece Automation with RFID Identifier Published Patent Application SK 50063-2016 A." Darina Matisková, Michal Balog, Pavol Semančo Banská Bystrica: IPO SR 7 (2017).

[12] El Abkari, Safae, and Jamal Mhamdi. "The nervous system modeling algorithm for signal processing and communication in intelligent networks." 2016 2nd International Conference on Advanced Technologies for Signal and Image Processing (ATSIP). IEEE, 2016. https:// doi.org/10.1109/atsip.2016.7523182

[13] Mhamdi, Jamal et El Abkari, Safae. Contriving an RFID system for Alzheimer patients tracking. In: 2015 Third International Workshop on RFID And Adaptive Wireless Sensor Networks (RAWSN). IEEE, 2015. p. 23-28. https://doi.org/10.1109/rawsn.2015.7173273

[14] Chang, Ching-Hsiang, Yeong-Lin Lai, and Chih-Cheng Chen. "Implement the RFID position-based system of automatic tablets packaging machine for patient safety." Journal of medical systems 36.6 (2012): 3463-3471. https://doi.org/10.1007/s10916-011-9799-6

[15] Koutkias, Vassilis G., et al. "A personalized framework for medication treatment management in chronic care." IEEE Transactions on Information Technology in Biomedicine 14.2 (2009): 464-472.

[16] Chien, Hung-Yu, et al. "Two RFID-based solutions to enhance inpatient medication safety." Journal of Medical Systems 35.3 (2011): 369-375. https://doi.org/10.1007/s10916-009 $\underline{-9373-7}$

[17] Chen, Mao, and Yong Lin. "Exploration and Implementation of Intelligent Park Information System based on Cloud Computing and Internet of Things." International Journal of Online and Biomedical Engineering (iJOE) 15.01 (2019): 115-128. https://doi.org/10.39 91/ijoe.v15i01.9783

[18] Yang, Jingjing, et al. "Indoor Positioning Method Based on Wireless Signal." International Journal of Online and Biomedical Engineering (iJOE) 14.10 (2018): 53-67.

[19] Yang, Jingjing, et al. "Indoor Positioning Method Based on Wireless Signal." International Journal of Online and Biomedical Engineering (iJOE) 14.10 (2018): 53-67.

[20] Koshy, Ranie. "Navigating the information technology highway: computer solutions to reduce errors and enhance patient safety." Transfusion 45 (2005): 189S-205S. https://doi.org/ $\underline{10.1111 / j .1537-2995.2005 .00619 . x}$

[21] Mirea, Adrian, and Adriana Albu. "Acquisition of physical data in an automated system for monitoring medication stocks." 2018 IEEE 12th International Symposium on Applied Computational Intelligence and Informatics (SACI). IEEE, 2018. https://doi.org/10.1109/ saci.2018.8440977

[22] El Abkari, Safae, Abdelilah Jilbab, and Jamal El Mhamdi. "Real time positioning over WSN and RFID network integration." 2018 4th International Conference on Advanced Technologies for Signal and Image Processing (ATSIP). IEEE, 2018. https://doi.org/10.11 $\underline{09 / a t s i p .2018 .8364443}$ 


\section{Authors}

Safae El Abkari is a Ph. D student. She completed her Electrical Engineering and Computer Science Bachelor degree in Ecole Normale Supérieure de l'Enseignement Technique of Rabat, Morocco in 2013. In 2015, she obtained her master degree of Electrical Engineering. She is a member of Electronic Systems, Sensors and Nanotechnologies laboratory (ENSET-Rabat) and Association of Signal and Image Technology (ATMS - Tunisia). Her interest in research motivates her to work hard to conceive and create interesting research projects. Her main expertise is computer Science, biomedical engineering system, and telecommunication. Her other interest includes software engineering, design and implementation system such as locating system with multiple technologies.

Abdelilah Jilbab is a professor at the Ecole Normale Supérieure de l'Enseignement Technique of Rabat, Morocco; he acquired a Computer and Telecommunication PhD from Mohamed V University, Rabat, Morocco in 2009. His thesis concerns the Filtering of illegal sites on the Internet: Contribution to the type of image recognition based on the principle of Maximum Entropy. He is a member of Electronic Systems, Sensors and Nanotechnologies laboratory (ENSET-Rabat) and member of LRIT laboratory (Unit associated with the CNRST, FSR, Mohammed V University, Rabat, Morocco) Since 2003. His domains of interest include embedded systems and signal processing.

Jamal EL Mhamdi is a professor at Ecole Normale Supérieure de l'Enseignement Technique of Rabat, Morocco. Major: Computer Science and Telecommunication. His thesis concerns Signal processing and telecommunications. He is a member of Electronic Systems, Sensors and Nanotechnologies laboratory (ENSET-Rabat). His interest in teaching motivates him to work hard in teaching, working on the conception of systems concreting his expertise in software Engineering, System design and telecommunication.

Article submitted 2020-07-14. Resubmitted 2020-08-08. Final acceptance 2020-08-10. Final version published as submitted by the authors. 